\title{
A SUFFICIENT CONDITION FOR AN ARC TO BE NEARLY POLYHEDRAL
}

\author{
JAMES M. MCPHERSON
}

\begin{abstract}
We give a simple geometric condition for an arc to be wild at an endpoint, with penetration index at least three.
\end{abstract}

0 . Proving that a given arc is "nearly polyhedral" (see [1]) is a very difficult task and, to the author's knowledge, has only been done twice in the published literature [1], [2]. The method used by Fox and Artin in [2] was algebraic, while the method used by Alford and Ball was purely geometric; no general set of sufficient conditions for an arc to be nearly polyhedral seems to be known.

We shall prove that an arc is nearly polyhedral if certain rather mild geometric conditions are satisfied. Since the $\operatorname{arcs} A_{n}$ of [1] and Example 1.2 of [2] satisfy these conditions, their wildness follows immediately.

This result is a generalization of a result in the author's 1970 doctoral thesis at The University of New South Wales, Australia. The author wishes to thank Professor N. F. Smythe for drawing his attention to a serious error in the original draft of this paper, and for suggesting the necessary correction.

1. Let $X$ be a set. We use $\operatorname{Bd} X, \mathrm{Cl} X$, and Int $X$ to denote the boundary, closure, and interior respectively of $X . N(X)$ is the number of points of $X$. If $k$ is an oriented arc in an oriented 3-manifold, and $X$ is an oriented surface, $\nu(k \cap X)$ is the algebraic intersection number of $k$ with $X$ (Schnittzahl- $[4, \$ \$ 69,70])$.

Let $L=l_{1} \cup l_{2}$ be a link of two components in Euclidean 3-space $E^{3}$. Then $L$ is splittable if there exists a 2 -sphere $S \subset E^{3}$ such that $l_{1}$ and $l_{2}$ lie in different components of $E^{3}-S$; otherwise $L$ is unsplittable. For $L$ to be unsplittable, it is sufficient that the linking number $\lambda\left(l_{1}, l_{2}\right)$ of $L$ be nonzero [4, p. 278].

Let $L=l_{1}^{\prime} \cup l_{2}^{\prime}$ be another link in $E^{3}$. Then $L^{\prime}$ arises from $L$ by a simple $F$-isotopy on the $i$ th component if $L-l_{i}=L^{\prime}-l_{i}^{\prime}$, and there is a solid torus $V \subset E^{3}-\left(L-l_{i}\right)$ such that either

(i) $l_{i}$ is a core of $V$, and $l_{i}^{\prime}$ has winding number one in $V$, or

(ii) $l_{i}^{\prime}$ is a core of $V$, and $l_{i}$ has winding number one in $V$.

Received by the editors April 29, 1970.

AMS 1969 subject classifications. Primary 5520, 5705; Secondary 0550.

Key words and phrases. Wild arc, nearly polyhedral arc, nice penetration index, "cutting and pasting". 
Two links are $F$-isotopic if one can be obtained from the other by a finite sequence of simple $F$-isotopies; $F$-isotopy includes polyhedral isotopy as a special case (cf. [5]).

If $k$ is an arc in $E^{3}$ which is tame except possibly at one endpoint $p$, say, then the (nice) penetration index $P(k, p)$ of $k$ at $p$ is the smallest integer $n$ such that there exist arbitrarily small closed 3-cell neighbourhoods of $p$, each one meeting $k$ on its boundary in $n$ points [3]. By Theorem V.1 of [3], we may assume that the closed 3-cells are tame.

Let $E_{i}$ and $E_{i+1}$ be tame closed 3-cell neighbourhoods of $p$, with $E_{i+1} \subset$ Int $E_{i} . A\left(E_{i}, E_{i+1}\right)$ denotes the set of arcs of $k$ in $E_{i}-E_{i+1}$ whose endpoints lie on $\mathrm{Bd} E_{i}$; i.e. the set of closed components of $k \cap\left(E_{i}-E_{i+1}\right)$. Similarly, $B\left(E_{i+1}, E_{i}\right)$ denotes the set of arcs of $k$ in $E_{i}-E_{i+1}$ whose endpoints lie on $\mathrm{Bd} E_{i+1}$, i.e. the set of closed components of $k \cap \operatorname{Int} E_{i} \cap \mathrm{Cl}\left(E^{3}-E_{i+1}\right)$.

For each $\alpha \in A\left(E_{i}, E_{i+1}\right)$ and $\beta \in B\left(E_{i+1}, E_{i}\right)$, there exist tame arcs $\alpha^{\prime} \subset \mathrm{Bd} E_{i}$ and $\beta^{\prime} \subset \mathrm{Bd} E_{i+1}$ such that $\alpha \cup \alpha^{\prime}$ and $\beta \cup \beta^{\prime}$ are tame simple closed curves. If $\alpha^{\prime \prime} \subset \mathrm{Bd} E_{i}$ and $\beta^{\prime \prime} \subset \mathrm{Bd} E_{i+1}$ are two other arcs joining the endpoints of $\alpha$ and $\beta$ respectively, then the links $\left(\alpha \cup \alpha^{\prime}\right) \cup\left(\beta \cup \beta^{\prime}\right)$ and $\left(\alpha \cup \alpha^{\prime \prime}\right) \cup\left(\beta \cup \beta^{\prime \prime}\right)$ are $F$-isotopic (in fact, they are isotopic by an ambient isotopy of $E^{3}$ which leaves $\alpha \cup \beta$ fixed). Consequently, $\left(\alpha \cup \alpha^{\prime \prime}\right) \cup\left(\beta \cup \beta^{\prime \prime}\right)$ is splittable iff $\left(\alpha \cup \alpha^{\prime}\right) \cup\left(\beta \cup \beta^{\prime}\right)$ is splittable, by Theorem 1 of [5]. Thus it makes sense to say that the pair $(\alpha, \beta)$ is splittable or unsplittable (with respect to the pair $\left.E_{i} \supset E_{i+1}\right)$ if there exists one link $\left(\alpha \cup \alpha^{\prime}\right) \cup\left(\beta \cup \beta^{\prime}\right)$ which is splittable or unsplittable.

2. For the pair $E_{i} \supset E_{i+1}$, consider the statements $(\mathrm{S} 1)_{i}$ and $(\mathrm{S} 2)_{i}$ given below.

$(\mathrm{S} 1)_{i}$. For each $\alpha \in A\left(E_{i}, E_{i+1}\right)$, there exists a $\beta \in B\left(E_{i+1}, E_{i}\right)$ such that the pair $(\alpha, \beta)$ is unsplittable.

$(\mathrm{S} 2)_{i}$. For each $\beta \in B\left(E_{i+1}, E_{i}\right)$, there exists an $\alpha \in A\left(E_{i}, E_{i+1}\right)$ such that the pair $(\alpha, \beta)$ is unsplittable.

TheOREM. Let $E_{0} \supset E_{1} \supset E_{2} \supset$. . be a sequence of tame closed 3-cell neighbourhoods of $p$, such that for each $i=0,1,2, \cdots$,

(i) the sets $A\left(E_{i}, E_{i+1}\right)$ and $B\left(E_{i+1}, E_{i}\right)$ are nonempty, and

(ii) the statements (S1) ${ }_{i}$ and $(\mathrm{S} 2)_{i}$ are true for the pair $E_{i} \supset E_{i+1}$. Then $P(k, p) \geqq 3$ (and $k$ is wild at $p$ ).

Proof. Keeping $E_{0}$ fixed, let $\mathcal{E}$ be the class of all sequences $E_{0}$ $=B_{0}^{\prime} \supset B_{1}^{\prime} \supset B_{2}^{\prime} \supset \cdots$ of tame closed 3 -cell neighbourhoods of $p$ satisfying conditions (i) and (ii) of the theorem. $\mathcal{E}$ is not empty, by hypothesis. 
Suppose $P(k, p)=1$, and choose a 3-cell $C \subset$ Int $E_{0}$ with $p \in \operatorname{Int} C$ and $N(k \cap \mathrm{Bd} C)=1$. By Theorem V.1 of [3], we may assume that $C$ is tame. Then there exists a sequence in $\mathcal{E}$, say $E_{0} \supset B_{1} \supset B_{2} \supset B_{3}$ $\supset \cdots$, with the following properties:

(i) there exist indices $h$ and $H$ such that $\operatorname{Bd} C \subset \operatorname{Int}\left(B_{h-1}-B_{H+1}\right)$, and $\mathrm{Bd} C$ is in general position with respect to $\mathrm{Bd} B_{h}, \mathrm{Bd} B_{h+1}, \cdots$, $\mathrm{Bd} B_{H}$, and

(ii) the number of intersection curves $\mathrm{Bd} C \cap \bigcup_{j=h}^{H} \mathrm{Bd} B_{j}$ is minimal amongst all the sequences in $\mathcal{E}$.

We call this sequence $B$.

The curves $\mathrm{Bd} C \cap \cup_{j=h}^{H} \mathrm{Bd} B_{j}$ are all null-homologous on Bd $C$. Therefore, there is an index $s$ and an intersection curve $\sigma \subset \mathrm{Bd} C$ $\cap \mathrm{Bd} B_{s}$ which bounds a disc $D \subset \mathrm{Bd} C$ containing no other intersection curves. $\sigma$ separates $\mathrm{Bd} B_{s}$ into two discs-one of these, $D^{\prime}$ say, together with $D$ is the boundary of a 3-cell $S$ which does not contain $B_{s+1}$.

Suppose $N\left(k \cap D^{\prime}\right)>N(k \cap D)$, i.e. there exists a subarc $\alpha$ of $k$ with $\alpha \cap \operatorname{Bd} B_{s} \subset$ Int $D^{\prime}$ and Int $\alpha \subset$ Int $S$. Then either $\alpha \in A\left(B_{s}, B_{s+1}\right)$ or $\alpha \in B\left(B_{s}, B_{s-1}\right)$, according as Int $D$ lies in $B_{s}-B_{s+1}$ or $B_{s-1}-B_{s}$.

We join the endpoints of $\alpha$ by an arc $\alpha^{\prime} \subset$ Int $D^{\prime}$; then if $\alpha \in$ $A\left(B_{s}, B_{s+1}\right)$, we let $\beta$ be any element of $B\left(B_{s+1}, B_{s}\right)$. Now because $B_{s+1} \subset \operatorname{Int}\left(E_{0}-S\right)$ and the endpoints of $\beta$ lie on $\mathrm{Bd} B_{s+1}, \nu(\beta \cap \mathrm{Bd} S)$ $=0$ (we have given $k$ an orientation, which induces an orientation on $\beta) . \nu(\beta \cap D)=0$ because $\beta \cap \mathrm{Bd} S=\beta \cap D$; but $N(\beta \cap D) \leqq$ $N(k \cap \operatorname{Bd} C)=1$, so $\beta$ cannot meet $D$ at all.

Then $\beta \cap \operatorname{Bd} S=\varnothing$ and $\beta \cap S=\varnothing$. By choosing a small enough closed regular neighbourhood $S^{\prime}$ of $S$, we obtain a 2 -sphere $\mathrm{Bd} S^{\prime}$ which splits the link $\left(\alpha \cup \alpha^{\prime}\right) \cup\left(\beta \cup \beta^{\prime}\right)$, where $\beta^{\prime} \subset \mathrm{Bd} B_{s+1}$ is any arc joining the endpoints of $\beta$. The pair $(\alpha, \beta)$ is therefore splittable; since this is true for each $\beta \in B\left(B_{s+1}, B_{s}\right)$, this contradicts hypothesis (S1). Therefore no such subarc $\alpha \in A\left(B_{s}, B_{s+1}\right)$ can exist.

Similarly, no such subarc $\alpha \in B\left(B_{s}, B_{s-1}\right)$ can exist without contradicting the hypothesis $(\mathrm{S} 2)_{s-1}$.

It follows then that $N\left(k \cap D^{\prime}\right) \leqq N(k \cap D) \leqq 1$. Neither endpoint of $k$ lies in $S$, so $\nu(k \cap \operatorname{Bd} S)=0$; thus $k \cap S$ is either empty, or is an arc joining the two points $k \cap D$ and $k \cap D^{\prime}$.

Let $N$ be a small closed regular neighbourhood of $S$. If $D \subset B_{8}$, write $B_{s}^{\prime}=\mathrm{Cl}\left(B_{s}-N\right)$, while $B_{s}^{\prime}=B_{s} \cup N$ if $D \subset \mathrm{Cl}\left(E_{0}-B_{s}\right)$. Then $\mathrm{Bd} C$ is in general position with respect to $\mathrm{Bd} B_{h}, \cdots, \mathrm{Bd} B_{s-1}$, $\mathrm{Bd} B_{s}^{\prime}, \mathrm{Bd} B_{s+1}, \cdots, \mathrm{Bd} B_{H}$, and

$$
\operatorname{Bd} C \cap\left(\operatorname{Bd} B_{s}^{\prime} \cup \bigcup_{j \neq s} \operatorname{Bd} B_{j}\right) \subset \operatorname{Bd} C \cap \bigcup_{j=h}^{H} \operatorname{Bd} B_{j}-\{\sigma\} .
$$


We need to show that the conditions $(\mathrm{S} 1)_{8-1},(\mathrm{~S} 2)_{8-1},(\mathrm{~S} 1)_{8}$ and (S2), are satisfied for $B_{s-1} \supset B_{s}^{\prime} \supset B_{s+1}$, so that the sequence $E_{0} \supset B_{1}$ $\supset \ldots \supset B_{s-1} \supset B_{s}^{\prime} \supset B_{s+1} \supset \ldots$ will be a sequence in $\mathcal{E}$, contradicting the minimality assumption involved in the choice of our sequence $B$. If $k \cap S=\varnothing$, there is nothing to prove, for then $A\left(B_{s}^{\prime}, B_{s+1}\right)=A\left(B_{s}, B_{s+1}\right)$ and $B\left(B_{s}^{\prime}, B_{s-1}\right)=B\left(B_{s}, B_{s-1}\right)$. So we suppose $k \cap S \neq \varnothing$.

$(\mathrm{S} 1)_{s-1}$. Let $\alpha \in A\left(B_{s-1}, B_{s}^{\prime}\right)=A\left(B_{s-1}, B_{s}\right)$, and let $\beta \in B\left(B_{s}, B_{s-1}\right)$ be chosen so that the pair $(\alpha, \beta)$ is unsplittable. $\beta$ is also an element of $B\left(B_{s}^{\prime}, B_{s-1}\right)$ unless one of the endpoints of $\beta$ lies in Int $D^{\prime}$. If this is the case, we join the endpoints of $\beta$ by an $\operatorname{arc} \beta^{\prime} \subset \mathrm{Bd} B_{s}$, so that $\beta^{\prime}$ meets the intersection curve $\sigma=\operatorname{Bd} D^{\prime}$ in exactly one point, say $x$. Let $\gamma$ be an arc joining the points $x$ and $k \cap D$, with Int $\gamma \subset$ Int $D$.

Let $\alpha^{\prime} \subset \mathrm{Bd} B_{s-1}$ be an arc joining the endpoints of $\alpha$, and let $\beta^{\prime \prime} \subset \mathrm{Bd} B_{s}^{\prime}$ be the arc $\gamma \cup\left(\beta^{\prime} \cap\left(\mathrm{Bd} B_{s}-D^{\prime}\right)\right)$. If $B_{s}^{\prime} \subset B_{s}, \quad\left(\alpha \cup \alpha^{\prime}\right)$ $\cup\left[\beta \cup(k \cap S) \cup \beta^{\prime \prime}\right]$ is $F$-isotopic to $\left(\alpha \cup \alpha^{\prime}\right) \cup\left(\beta \cup \beta^{\prime}\right)$ by a simple $F$-isotopy on the second component-indeed, let the solid torus $V$ be a closed regular neighbourhood of $\beta \cup S$. By Theorem 1 of [5], then, the pair $(\alpha, \beta \cup(k \cap S))$ is unsplittable because the pair $(\alpha, \beta)$ is unsplittable. We note that $\beta \cup(k \cap S) \in B\left(B_{s}^{\prime}, B_{s-1}\right)$.

If $B_{s} \subset B_{s}^{\prime}$, the links $\left(\alpha \cup \alpha^{\prime}\right) \cup\left[\left(\beta \cap\left(E_{0}-B_{s}^{\prime}\right)\right) \cup \beta^{\prime \prime}\right]$ and $\left(\alpha \cup \alpha^{\prime}\right)$ $\cup\left(\beta \cup \beta^{\prime}\right)$ are $F$-isotopic; so the pair $\left(\alpha, \beta \cap \mathrm{Cl}\left(E_{0}-B_{s}^{\prime}\right)\right)$ is unsplittable. We note that $\beta \cap \mathrm{Cl}\left(E_{0}-B_{s}^{\prime}\right)$ is an element of $B\left(B_{s}^{\prime}, B_{s-1}\right)$.

In both cases, then, there exists a $\beta^{*} \in B\left(B_{s}^{\prime}, B_{s-1}\right)$ such that the pair $\left(\alpha, \beta^{*}\right)$ is unsplittable. Since this is true for each $\alpha \in A\left(B_{s-1}, B_{s}^{\prime}\right)$, the pair $B_{s-1} \supset B_{s}^{\prime}$ satisfies the hypothesis $(\mathrm{S} 1)_{s-1}$.

That $(\mathrm{S} 2)_{s-1},(\mathrm{~S} 1)_{s}$ and $(\mathrm{S} 2)_{s}$ are also satisfied requires a similar proof, which is omitted.

Then the sequence $E_{0} \supset B_{1} \supset B_{2} \supset \cdots \supset B_{s-1} \supset B_{s}^{\prime} \supset B_{s+1} \supset \cdots$ is a sequence in $\mathcal{E}$ which meets $\mathrm{Bd} C$ on its boundary surfaces in fewer curves than does our sequence $B$, so we must assume that $\mathrm{Bd} C \cap$ UBd $B_{j}=\varnothing$. That is, $\mathrm{Bd} C$ meets none of the surfaces $\mathrm{Bd} B_{1}$, Bd $B_{2}, \cdots$.

Hence there exists an index $j$ such that $B d C \subset \operatorname{Int}\left(B_{j}-B_{j+1}\right)$. Since $N(k \cap \operatorname{Bd} C)=1, \alpha \cap \operatorname{Bd} C=\varnothing$ if $\alpha \in A\left(B_{j}, B_{j+1}\right)$, because both endpoints of $\alpha$ lie on $\operatorname{Bd} B_{j} C \operatorname{Int}\left(E_{0}-C\right)$. Similarly, $\beta \cap \operatorname{Bd} C=\varnothing$ for all $\beta \in B\left(B_{j+1}, B_{j}\right)$.

But then the pair $(\alpha, \beta)$ is splittable for each $\alpha \in A\left(B_{j}, B_{j+1}\right)$ and $\beta \in B\left(B_{j+1}, B_{j}\right)$, because $\alpha \cup \operatorname{Bd} B_{j}$ and $\beta \cup B_{j+1}$ lie in different components of $E^{3}-\mathrm{Bd} C$. This contradicts the hypotheses $(\mathrm{S} 1)_{j}$ and $(\mathrm{S} 2)_{j}$.

Because $(\mathrm{S} 1)_{j}$ and $(\mathrm{S} 2)_{j}$ are true for all values of $j$, it is impossible 
that there exist a closed 3-cell $C$ with $N(k \cap \mathrm{Bd} C)=1$ and $\mathrm{Bd} C$ $C \operatorname{Int}\left(B_{j}-B_{j+1}\right)$ for any value of $j$. Therefore no 3-cell $C \subset$ Int $E_{0}$ can exist with $N(k \cap \mathrm{Bd} C)=1$; that is, $N(k \cap \mathrm{Bd} C) \geqq 3$ and $P(k, p)$ $\geqq 3$. Q.E.D.

\section{REFERENCES}

1. W. R. Alford and B. J. Ball, Some almost polyhedral wild arcs, Duke Math. J. 30 (1963), 33-38. MR 26 \#1858.

2. R. H. Fox and E. Artin, Some wild cells and spheres in three-dimensional space, Ann. of Math. (2) 49 (1948), 979-990. MR 10, 317.

3. D. W. Henderson, Self-unlinked simple closed curves, Trans. Amer. Math. Soc. 120 (1965), 470-480. MR $32 \# 4687$.

4. H. Seifert and W. Threlfall, Lehrbuch der Topologie, Teubner, Leipzig, 1934; reprint, Chelsea, New York, 1947.

5. N. Smythe, Topological invariants of isotopy of links. I, Amer. J. Math. 92 (1970), 86-98.

The University of New South Wales, Kensington, New South Wales, Australia 